Topiques, études satoriennes

Topoï Studies, Journal of the SATOR

\section{Bibliographie générale}

Volume 5, 2021

URI: https://id.erudit.org/iderudit/1081529ar

DOI: https://doi.org/10.7202/1081529ar

See table of contents

Publisher(s)

SATOR, Société d'Analyse de la Topique Romanesque d'Ancien Régime

ISSN

2369-4831 (digital)

Explore this journal

Cite this document

(2021). Bibliographie générale. Topiques, études satoriennes / Topoï Studies, Journal of the SATOR, 5, 169-178. https://doi.org/10.7202/1081529ar

$\begin{array}{lllll}\mathbf{S} & \mathbf{A} & \mathbf{T} & \boldsymbol{\odot} & \mathbf{R} \\ \mathbf{A} & R & E & P & O \\ \mathbf{T} & E & N & E & T \\ \boldsymbol{0} & P & E & R & A \\ \mathbf{R} & \bigcirc & T & A & S\end{array}$




\section{BIBLIOGRAPHIE GÉNÉRALE}

CAMUS, Jean-Pierre, Les spectacles d'horreur, Paris, Classiques Garnier, 2013, [S. Ferrari (éd.)]. DULAURE, Jacques Antoine, Histoire critique de la noblesse, depuis le commencement de la monarchie jusqu'à nos jours; où l'on expose ses préjugés, ses brigandages, ses crimes, où l'on prouve qu'elle a été le fléau de la liberté, de la raison, des connoissances humaines, et constamment l'ennemi du peuple et des rois, Paris, Guillot imprimeur-libraire, 1790.

JAKEMÉS, Le livre des amours du Chastellain de Coucy et de la Dame de Fayel, Lille, Presses universitaires de Lille, 1994, [A. Petit et F. Suard (éds.)].

JAKEMÉS, Le roman du châtelain de Coucy et de la dame de Fayel, Troësnes, Corps 9, 1986 [A. Petit et F. Suard (trad.)].

JAKEMÉS, Le roman du châtelain de Coucy et de la dame de Fayel (éd. bilingue), Paris, Honoré Champion, 2009, [C. Gaullier-Bougassas (trad. / ed.)].

MARY, André (trad.), La chambre des dames : contes et romans du Moyen Âge, Paris, Gallimard, 1943.

LUSSAN, Marguerite de, Anecdotes de la cour de Philippe-Auguste, Amsterdam, Jean Pauli et la veuve de P. De Coup, 1733.

RENAUD, de Beaujeu, Le lai de l'Ignauré, ou, Lai du prisonnier, Liège, Vaillant-Carmanne (Textes anciens, Académie royale de langue et de littérature françaises de Belgique), 1938, t. 3, [R. Lejeune-Dehousse (ed.)].

SADE, Donatien Alphonse François marquis de, Euvres compètes du Marquis de Sade, Paris, Pauvert, 1986, 15 t. [A. Le Brun et J.-J. Pauvert (eds.)].

COMBES, Annie, « Entre déférence et différence, les ambiguïtés de la mise en prose dans Le livre des amours du Chastellain de Coucy et de la Dame de Fayel », D. Kullmann et S. Lalonde, Réécritures : Regards nouveaux sur la reprise et le remaniement de textes dans la littérature française et au-delà, du Moyen Âge à la Renaissance, Toronto, Pontifical Institute of Mediaeval Studies, 2015, p. 53-72.

DENIS, Françoise, « Cœur arraché / Cœur mangé : modulations », Études littéraires, vol. 31, nº 1, 1998, p. 95-108.

DI FEBO, Martina, «Ignauré. La parodie "dialectique" ou le détournement du symbolisme courtois », Cahiers de recherches médiévales [En ligne], 5 | 1998, mis en ligne le 01 octobre 2007, consulté le 01 octobre 2016. URL : http://crm.revues.org/1482 ; DOI : 10.4000/ crm.1482.

DI MAIO, Mariella, Le cour mangé : Histoire d'un thème littéraire du Moyen Âge au XIXe siècle, Paris, Presses de l'université Paris-Sorbonne, 2005.

HAUVETTE, Henri, « La 39 nouvelle du Décaméron et la légende du “Cœur mangé" », Romania, t. $41, \mathrm{n}^{\circ} 162,1912$, p. $184-205$.

HENEIN, Eglal, « Le cœur mangé... ruminé », Martine DEBAISIEUX et Gabrielle VERDIER (eds.), Violence et fiction jusqu'à la Révolution, Tübingen, Gunter Narr Verlag, 1998, p. 77-88.

JEAY, Madeleine, "La cruauté de Philomèle : métamorphoses médiévales du mythe ovidien », dans Martine DEBAISIEUX et Gabrielle VERDIER (eds.), Violence et fiction jusqu'à la Révolution, Tübingen, Gunter Narr Verlag, 1998, p. 111-119.

PASCAL, Jean-Noël, Le cour terrible, Perpignan, Presses universitaires de Perpignan, 2005.

RÄKEL, Hans-Herbert S., "Castelain de Couci», MGG. Die Musik in Geschichte und Gegenwart : allgemeine Enzyklopädie der Musik, Personenteil 4, Kassel, Bärenreiter ; Stuttgart, Metzler, 1994, T. 2, P. 4., p. 775-781.

Topiques, Études satoriennes, 5, 2020, Le Boire et le manger, https://journals.uvic.ca/index.php/sator/index 
RIBÉMONT, Bernard, «"Le cuer del ventre li as trais” : cœur arraché, cœur mangé, cœur envolé : un regard médico-théologique sur quelques thèmes littéraires », Le "cuer" au Moyen Âge : (réalité et senefiance), Aix-en-Provence, C.U.E.R.M.A. (Senefiance), 1991, nº 30, p. 347-361.

VICENSINI, Jean-Jacques, « Figure de l'imaginaire et figure du discours. Le motif du "cœur mangé" dans la narration médiévale », Le "cuer" au Moyen Age : (réalité et senefiance), Aix-enProvence, C.U.E.R.M.A. (Senefiance), 1991, n 30, p. 441-459.

Anonyme, Lazarillo de Tormes (1554), version digitale de wikisource consultée le 20 septembre 2017 : https://es.wikisource.org/wiki/E1_Lazarillo de Tormes.

BARRA JOVER, M., “Razón de amor”. Texte critique et composition, dans Revista de Literatura Medieval, I (1989); p. 123-53, vv. 239-42.

CERVANTES, Miguel de, Don Quijote de la Mancha, II ${ }^{\mathrm{e}}$ Partie, chap. 51. https://cvc.cervantes.es/literatura/clasicos/quijote/edicion/parte2/cap51/cap51 03.htm. Consulté le 10 août 2017.

FEIJOO, Jerónimo Benito, Teatro crítico universal, (1726-1740), Madrid, Joaquín Ibarra, rééd. 1775 , t. I.

IGLESIAS DE LA CASA, José, «Anacreóntica X», Poesías póstumas 1793-1798, dans Leopoldo Augusto DE CUETO (ed.), Biblioteca de autores españoles, t. I, «Poetas líricos del siglo XVIII », Madrid, Rivadeneyra impr., 1869.

ISAMBERT, DECRUSY, JOURDAN, TAILLANDIER, Recueil général des anciennes lois françaises depuis l'an 420 jusqu'à la révolution de 1789, 1514-1746, t. XII, Paris, Chez Belin-LePrieur Paris, 1822-23.

MEÏR ZABARA, Joseph ben (Juif catalan de fin du XII ${ }^{\mathrm{e}}$ siècle), trad. anglaise par Israel Abrahams, The Book of Delight, 1912 : www.gutenberg.org/ebooks/9886. Consulté pour la dernière fois le 13 septembre 2018.

ROJAS, Fernando de, Tragicomedia de Calixto y Melibea (1499), éd. digitale consultée le 20 septembre 2017: https://es.wikisource.org/wiki/La_Celestina.

RUIZ, Juan, Archiprêtre de Hita, Libro de Buen Amor (1330), Madrid, Espasa-Calpe, 1974 [J. Joset (ed.)].

MÜLLER, M. J., Sitzunsberichte der königlich Bayerischen Akademie der Wissenschaften, Munich, 1860.

Anonyme, As Obras de Gil Vicente, Lisboa, Imprensa Nacional, Casa da Moeda, Lisboa, Universidade de Lisboa, Centro de Estudos de Teatro, 5 vols., 2002 [José Camões José (ed.)].

Cantigas D'Escarnho e de Mal Dizer dos Cancioneiros Medievais Galego-Portugueses, Editorial Galaxia, 1965 [Manuel Rodrigues Lapa (ed.)].

QUEIRÓS, Eça de, A Cidade e as Serras, Lisboa, Livros do Brasil, s.d.

QUEIRÓS, Eça de, A Relíquia, Lisboa, Livros do Brasil, s.d. [Helena Cidade Moura (ed.)].

QUEIRÓS, Eça de, "Frei Genebro," Contos, Lisboa, Livros do Brasil, s.d., p. 107-120.

QUEIRÓS, Eça de, “O Francesismo,” Últimas Páginas, Obras de Eça de Queiroz,

Vol. II, Porto, Lello \& Irmão Editores, s.d., p. 813-827.

QUEIRÓS, Eça de, O Primo Basílio, Lisboa, Livros do Brasil, s.d., [Helena Cidade Moura (ed.)].

QUEIRÓS, Eça de, A Capital, Editorial Presença, 2003.

QUEIRÓS, Eça de, Textos de Imprensa, IV da Gazeta de Noticias, Lisboa, INCM, 2002 [Elza Miné e Neuma Cavalcante (ed.)].

QUEIRÓS, Eça de, O Crime do Padre Amaro, Lisboa, INCM, 2000 [Carlos Reis e Maria do Rosário Cunha (ed.)].

Topiques, Études satoriennes, 5, 2020, Le Boire et le manger, https://journals.uvic.ca/index.php/sator/index 
QUEIROZ, Eça de, The City and The Mountains, Manchester, Carcanet in association with the Calouste Gulbenkian Foundation, 1994 [R. Campbell (trans.)].

QUEIRÓS, Eça de, O Mandarim, Lisboa, INCM, 1992 [B. Berrini (ed.)].

QUEIRÓS, Eça de, Correspondência, Lisboa, INCM, 1983, vol.1.

QUEIRÓS, Eça de, Páginas de Jornalismo, Porto, Lello \& Irmão Editores, 1981 [A. Pinto de Castro (ed.)].

QUEIRÓS, Eça de, Os Maias. Episódios da vida romântica, Lisboa, Livros do Brasil, 1888.

RESENDE, Garcia de, Vida e feitos d'El-Rey Dom João II, Coimbra, Centro de Estudos de Linguística Geral e Aplicada (CELGA), Faculdade de Letras, Universidade de Coimbra, 2007 [E. Verdelho (ed.)].

RESENDE, Garcia de, Crónica de Dom João II e miscelânea, Imprensa Nacional Casa da Moeda, 1973 [J. V. Serrão (ed.)].

SILVA, António Manuel Policarpo da, O piolho viajante. Divididas as viagens em mil e uma carapuças, Lisboa, Estúdios Cor, 1973.

TAVARES, Paulino Mota e PEREIRA, Ana Mafalda, Descoberta e Invenção do Brasil-O amargo e o doce, Sintra, Colares Editora, 2000.

VICENTE, Gil, Auto da Festa: obra desconhecida com uma explicação prévia pelo Conde de Sabugosa, Lisboa, Imprensa Nacional, 1906.

VICENTE, Gil, Tragicomedia Pastoril da Serra da Estrella. Four plays of Gil Vicente, Coimbra, Atlântida Coimbra, 1963.

VICENTE, Gil, Obras completas, [Álvaro Júlio da Costa Pimpão (coord.)], Porto, Livraria Civilização, 1979.

ZURARA, Gomes Eanes de, Crónica da tomada de Ceuta por el-rei D. João I, Lisboa, Academia das Ciências (Série vermelha, $\mathrm{n}^{\circ}$ 1767), 1915.

GOES, Maria Antónia, À mesa com Eça de Queirós, Sintra, Colares Editora, 2004.

PALLA, Maria José "Cozinhar e contar uma história: o imaginário alimentar em Gil Vicente", Actas do Quinto Congresso (1-8 Set. 1996), Oxford, Oxford University, Associação Internacional de Lusitanistas, 1998.

PEIXINHO, Ana Teresa, "Food aesthetics: culinary notes on the work of Eça de Queirós," in Joaquim PINHEIRO, Carmen SOARES (eds.), Food Heritages on Both Sides of the Sea, Coimbra, Coimbra University Press / Annablume, 2016, p. 199-218.

QUITÉRIO, José, Histórias e curiosidades gastronómicas, Lisboa, Assírio e Alvim, 1992.

ÁLVAREZ MIRAVAL, Blas, Libro intitulado La conservación de la salud del cuerpo y del alma, para el buen regimiento de la salud y más larga vida de la Alteza del sereníssimo Príncipe don Philippo nuestro señor [1597], Medina del Campo, Santiago del Canto, 1597.

ALZIEU, Pierre, JAMMES, Robert et LISSORGUES, Yvan, Floresta de poesías eróticas del Siglo de Oro, Toulouse, France Ibérie Recherche, Université de Toulouse-Le Mirail, 1975.

CERVANTES, Miguel de, Don Quijote de la Mancha, éd. Franscico Rico, Madrid, Punto de lectura, 2007Paris, Seuil, 1997 [A. Schulman (trad.)].

COVARRUBIAS, Sebastián de, Tesoro de la lengua castellana o española [1611], éd. Martín de Riquer, Barcelona, Alta Fulla, 1987.

Entremés primero de Melisendra, in Emilio Cotarelo y Mori, Colección de entremeses, loas, bailes, jácaras y mojigangas desde fines del XVI a mediados del XVIII, I, Madrid, Bailly Baillière, 1911, p. $105^{\mathrm{a}}$.

GÓMEZ DE TOLEDO, Gaspar, Tercera parte de la tragicomedia de Celestina [1536], Philadelphia, University of Pennsylvania, 1966 [M. E. Barrick (ed.)].

Topiques, Études satoriennes, 5, 2020, Le Boire et le manger, https://journals.uvic.ca/index.php/sator/index 
GRANADO MALDONADO, Diego, Libro de cozina en el cual se contiene el modo de guisar de comer en qualquier tiempo assi de carne como de pescado para sanos y enfermos, $y$ convalescientes: assi de pasteles, tortas y salsas, como de conservas a la usança española, italiana y tudesca de nuestros tiempos, Madrid, Luis Sánchez, 1599.

GRANADO MALDONADO, Diego, Libro del arte de cocina, Lleida, Pagès Editors, 1990.

LEÓN, Fray Luis de, De los nombres de Cristo, éd. Cristóbal Cuevas, Madrid, Cátedra, 1984.

MILAN, Luis, El cortesano, Madrid, sucesores de Rivadeneyra, 1874.

MINSHEU, John, Diálogos muy apazibles [1599], in « Diálogos de antaño », Revue Hispanique, XLV, n¹07, 1919.

NOLA, Roberto de, Libro de guisados, Tolède, Ramón de Petras, 1525. Éd. moderne utilisée : Le livre de cuisine / Libro de guisados, Paris, Classiques Garnier, 2011 [N. Peyrebonne (de. / trad.)]. PLAUTE, Menaechmi, in Comédies, IV, Paris, Les Belles Lettres, 1992, p. 8-85.

RODRÍGUEZ FLORIÁN, Juan, Comedia Florinea [1554], in Marcelino Menéndez y Pelayo, Orígenes de la novela, t. III, Madrid, Bailly Baillière, 1910, p. 157-311.

RUEDA, Lope de, El deleitoso. Compendio llamado el Deleytoso, en el qual se contienen muchos passos graciosos... [1567], in Obras de Lope de Rueda, t. II, Madrid, Real Academia Española, 1908.

TIMONEDA, Juan de, Comedia de los Meñenos, in Las tres comedias de Juan Timoneda [1559], reproduction fac-similée de l'éd. de 1559, Madrid, Real Academia Española, Tipografía de Archivos, 1936, Escena Primera, pages non numérotées.

VALDÉS, Juan de, Diálogo de la lengua, Madrid, Cátedra, 198 [C. Barbolani (ed.)].

CAMERA DE ASARTA, Vittorio, "Consideraciones sobre un punto dudoso del Quijote», Revista de filología española, XLVI, 1963, p. 179-180.

CARRO CARBAJAL, Eva Belén, «Saraos, juegos y ensaladas a lo divino : aportaciones al estudio de la literatura popular impresa del siglo XVI », in Natalia Fernández Rodríguez, María Fernández Ferreiro, Literatura medieval y renacentista en España: líneas y pautas, Universidad de Salamanca, Sociedad de Estudios Medievales y Renacentistas, 2012, p. 419-431.

JEANNERET, Michel, Des mets et des mots. Banquets et propos de table à la Renaissance, Paris, José Corti, 1987.

MÁRQUEZ VILLANUEVA, Francisco, « Teófilo Folengo y Cervantes », dans Fuentes literarias cervantinas, Madrid, Gredos, coll. «Biblioteca Románica Hispánica. II. Estudios y Ensayos », 1973, p. 258-358.

NADEAU, Carolyn, Food Matters: Alonso Quijano's Diet and the Discourse of Food in Early Modern Spain, Toronto, University of Toronto Press, 2016.

PEYREBONNE, Nathalie, La table et les aliments dans les dialogues et le théâtre du XVI siècle espagnol, Villeneuve d'Ascq, Presses Universitaires du Septentrion, 1998.

VARELA MERINO, Elena, Los galicismos en el español de los siglos XVI y XVII, vol. 1, Madrid, Consejo Superior de Investigaciones Científicas, 2009.

VON GEMMINGEN, Barbara, «Estudios lexicológicos sobre la lengua culinaria del siglo de oro "), Revue de linguistique romane, 59, 1995, p. 401-417.

FURETIÈRE, Antoine, Dictionnaire universel, Genève, Slatkine Reprints, 1972. (Réimpression de l'édition de La Haye-Rotterdam, 1690).

MONTAIGNE, Michel de, Essais, Livre Premier, Ed. Pierre Michel, Paris, Gallimard, 1965. SOREL, Charles, La Bibliothèque françoise, $2^{\text {nd }}$ édition, 1667, Genève, Slatkine Reprints, 1970. SOREL, Charles, De la Perfection de l'homme, Paris, Robert Le Nain, 1655.

Topiques, Études satoriennes, 5, 2020, Le Boire et le manger, https://journals.uvic.ca/index.php/sator/index 
SOREL, Charles, Histoire comique de Francion, In Romanciers du XVIIe siècle, éd. Antoine Adam, Paris, Gallimard (Bibliothèque de la Pléiade), 1958.

Viau, Théophile de, Première Journée. Euvres complètes (II), éd. Guido Saba, Paris, Honoré Champion, 1999. pp. 9-30.

ASSAF, Francis, « Francion, une étude carnavalesque », Littératures classiques, 41, 2001, pp. 8595.

BAKHTINE, Mikhaïl, L'œuvre de François Rabelais et la culture populaire au Moyen Age et sous la Renaissance, Paris, Gallimard, 1970.

DANDREY, Patrick, Le premier Francion de Charles Sorel, ou le «jeu du roman», Paris, Klincksieck, 2001.

DEBAISIEUX, Martine, «Francion ou la libre pensée travestie », Neophilologus, 72, 1988, pp. 191-99.

DEBAISIEUX, Martine, Le Procès du Roman: Ecriture et contrefaçon chez Charles Sorel, Saratoga, Anma Libri, Stanford French and Italian Studies, 1989. Republication, Éditions Paradigme, Orléans, France, 2000.

DE VOS, Wim, Le singe au miroir : Emprunt textuel et écriture savante dans les romans comiques de Charles Sorel, Tübingen, Gunter Narr Verlag, 1994.

FLANDRIN, Jean-Louis, «Alimentation et médecine. Histoire de l'alimentation occidentale » http://www.lemangeur-ocha.com/texte/alimentation-et-medecine/; mis en ligne 28/04/2003

FRICHE, François, Entre Terre et Ciel: Romans comiques et mystère de l'Incarnation (16201660), Paris, Hermann, 2017.

GODARD DE DONVILLE, Louise, Le Libertin des origines à 1665 : un produit des apologètes, Paris-Seattle-Tübingen, Biblio 17 (51), 1989.

GARAVINI, Fausta, "Francion rivisitato : Diacronia d'una struttura », Saggie et ricerche di letteratura francese, XIV, 1975, pp. 39-107

GARAVINI, Fausta, La Maison des Jeux : Science du roman et roman de la science au XVII siècle, Paris, Champion, 1998.

GÉLIS, Jacques, «L'individualisation de l'enfant », Histoire de la vie privée, 3, De la Renaissance aux Lumières, Ed. Philippe Ariès et Georges Duby, Paris, Seuil, 1986, pp. 311-31.

HODGSON, Richard G., « De la 'comédie humaine' à la 'perfection de l'homme' : Charles Sorel moraliste », in Charles Sorel polygraphe, éd. Emmanuel Bury et Eric Van der Schueren, Laval, Presses de l'Université Laval, 2006, pp. 19-30.

HOWELLS, Robin, Carnival to Classicism : The Comic Novels of Charles Sorel, Paris-SeattleTübingen, Biblio 17 (48), 1989.

Jeanneret, Michel, Des Mets et des mots : Banquets et propos de table à la Renaissance, Paris, Corti, 1987.

MORGANTE, Jole, «La réécriture de la première partie du Francion: techniques d'écriture libertine », Littératures Classiques, 41, 2001, pp. 14-30.

ROSELLINI, Michèle, "La fête libertine du Francion : du festin au banquet philosophique ", Cahiers textuels, "Charles Sorel, Histoire comique de Francion », 22, 2000, pp. 67-82.

ROUX, Olivier, Charles Sorel : La figure, la ligne et l'invention de l'auteur, Paris, Champion, 2014.

La Bible, Paris, 1990, Robert Laffont (Bouquins) [Ph. Sellier (eds.) / L.-I. Lemaître de Sacy (trad.)]. 
BOSSUET, Jacques Bénigne, Premier Panégyrique de saint Gorgon, dans Euvres oratoires de Bossuet, Bruges-Paris, Desclée-De Brouwer et Cie 1926, vol. 1, p. 31-46 [J. Lebarcq, Ch. Urbain et E. Levesque (eds.)].

BOSSUET, Jacques Bénigne, Second Panégyrique de saint Gorgon, dans CEuvres oratoires de Bossuet, Bruges-Paris, Desclée-De Brouwer et Cie, 1926, vol. 1, p. 576-586 [J. Lebarcq, Ch. Urbain et E. Levesque (eds.)].

BOSSUET, Jacques Bénigne, Sermon sur l'éminente dignité des pauvres dans l'Église, dans Euvres oratoires de Bossuet, Bruges-Paris, Desclée-De Brouwer et Cie, 1927, vol. 3, p. 119-138 [J. Lebarcq, Ch. Urbain et E. Levesque (eds.)].

BOSSUET, Jacques Bénigne, Pensées chrétiennes et morales, dans Euvres oratoires de Bossuet, Bruges-Paris, Desclée-De Brouwer et Cie, 1926, vol. 6, p. 640-707 [J. Lebarcq, Ch. Urbain et E. Levesque (eds.)].

LONGIN, Traité du sublime, Paris, Librairie Générale Française, 1995 [F. Goyet (eds.) / Boileau (trad.)]

VORAGINE, Jacques de, La Légende dorée, Paris, Garnier-Flammarion, 1967, t. 2 [Révérend Père H. Savon (eds.) / J.-B. M. Roze (trad.)].

BARTHES, Roland, S/Z, Paris, Seuil (Points), 1970.

BAKHTINE, Mikhaïl, L'Euvre de François Rabelais et la culture populaire au Moyen Âge et sous la Renaissance, Paris, Gallimard (Tel), 1970 [A. Robel (trad.)].

BIET, Christian (dir.), Théâtre de la cruauté et récits sanglants en France (XVI'-XVII siècle), Paris, Robert Laffont, (Bouquins), 2006.

CALVET, Jean, Bossuet, Paris, Hatier (Connaissance des lettres), 1968.

DOUGLAS, Mary, De la Souillure. Essai sur les notions de pollution et de tabou, Paris, La Découverte (Poche), 2005 [A. Guérin (trad.)].

JOLLES, André, Formes simples, Paris, Seuil (Poétique), 1972 [A. M. Buguet (trad.)].

MINOIS, Georges, Bossuet. Entre Dieu et le Soleil, Paris, Perrin, 2003.

WEILL, Michèle, RODRIGUEZ, Pierre, «Objectifs littéraires de la Sator », https://satorbase.org/index.php?do=outils\#objectifs, page consultée le 12/05/19.

BIBLIOGRAPHIE

Mercure galant, novembre 1711.

Anonyme, Le Petit-fils d'Hercule dans Romanciers libertins du XVIII siècle, t. II, Paris, Gallimard (Bibliothèque de la Pléiade), 2005, [P. Wald-Lasowski (éd.)], p. 1073-1137.

Anonyme, Les Mémoires de Suzon dans Romanciers libertins du XVIII siècle, t. II, Paris, Gallimard (Bibliothèque de la Pléiade), 2005, [P. Wald-Lasowski (éd.)] p. 873-971.

AULNOY (d'), Relation du voyage d'Espagne, Paris, chez Claude Barbin, 1691, 3 vol.

BEAUMARCHAIS, Mémoires contre M. Goëzmann, Euvres complètes, t. 3, Burne, [1774] 1828.

BERNARDIN DE SAINT-PIERRE, Voyage à l'île de France, Euvres posthumes, t. 1, Paris, Ledentu, 1840.

DIDEROT, Denis, La Religieuse dans Contes et romans, Paris, Gallimard (Bibliothèque de la Pléiade), 2004, [M. Delon (éd.)], p. 239-415.

DIDEROT, Denis, Lettre sur les sourds et muets à l'usage de ceux qui entendent et qui parlent dans Euvres complètes, t. IV, Le nouveau Socrate, Idées II, Paris, Hermann, 1978.

DIDEROT, Denis et LE ROND D’ALEMBERT, Jean (éds.), Encyclopédie, ou dictionnaire raisonné des sciences, des arts et des métiers, University of Chicago, ARTFL Encyclopédie Project [R. Morrissey et G. Roe (éds.)], automne 2017.

URL : http://encyclopedie.uchicago.edu/.

Topiques, Études satoriennes, 5, 2020, Le Boire et le manger, https://journals.uvic.ca/index.php/sator/index 
DU LAURENS, Le Compère Mathieu ou les Bigarrures de l'esprit humain, Paris, Honoré Champion, 2012 [D. Gambert (éd.)].

FOUGERET DE MONBRON, Margot la ravaudeuse [1750] dans Romanciers libertins $d u$ XVIII siècle, t. I, Paris, Gallimard (Bibliothèque de la Pléiade), 2000, [P. Wald-Lasowski (éd.)], p. 801-866.

GODARD D'AUCOUR, Thémidore dans Romanciers libertins du XVIII siècle, t. I, Paris, Gallimard (Bibliothèque de la Pléiade), 2000, [P. Wald-Lasowski (éd.)], p. 501-586.

GOULIN, Jean et LABEYRIE, (prénom non indiqué), Dictionnaire raisonné universel de matière médicale, Paris, chez François Didot, 1773, 4 vol.

LÉMERY, Nicolas, Traité universel des drogues simples, Paris, chez Laurent d'Houry, $3^{\mathrm{e}}$ édition, 1723.

LÉMERY, Louis, Traité des aliments où l'on trouve par ordre et séparément la différence et le choix qu'on doit faire de chacun d'eux en particulier, les bons et les mauvais effets qu'ils peuvent produire, les principes en quoi ils abondent..., Paris, J.-B. Cusson \& P. Witte, 1702, in-8 ; $2^{\mathrm{e}}$ éd., Paris, P. Witte, 1705.

LESAGE, Le Diable boiteux dans Romanciers du XVIII siècle, t. I, Paris, Gallimard (Bibliothèque de la Pléiade), [1726] 1987, [Étiemble (éd.)], p. 269-494.

LESAGE, Histoire de Gil Blas de Santillane dans Romanciers du XVIII siècle, t. I, Paris, Gallimard (Bibliothèque de la Pléiade), 1987, [Étiemble (éd.)], p. 495-1201.

LESAGE, Histoire de Guzman d'Alfarache, Berquet, 1825, 4 vol.

LOUVET DE COUVRAY, Six semaines de la vie de Faublas dans Romanciers du XVIII siècle, t. II, Paris, Gallimard (Bibliothèque de la Pléiade), 1965, [Étiemble (éd.)], p. 721-856.

MIRABEAU, Le Libertin de qualité, ou Ma conversion, Paris, Bibliothèque des Curieux, [1783] 1911.

NERCIAT, Félicia ou Mes fredaines dans Romanciers libertins du XVIII siècle, t. II, Paris, Gallimard (Bibliothèque de la Pléiade), [1775] 2005, [P. Wald-Lasowski (éd.)], p. 591-872.

POMET, Pierre, Histoire générale des drogues traitant des plantes, des animaux \& des Mineraux, ouvrage enrichy de plus de quatre cent Figures en Taille-douce tirées d'après Nature; avec un discours qui explique leurs differens Noms, les Pays d'où elles viennent, la maniere de connoître les Veritables d'avec les Falsifiées, \& leurs proprietez, où l'on découvre l'erreur des Anciens \& des Modernes ; Le tout très utile au Public, Paris, chez Estienne Ducastin, 1694.

RESTIF DE LA BRETONNE, L'Anti-Justine, Paris, Le Cercle Poche, [1798] 2014.

SADE, Les Infortunes de la vertu, Justine ou les Malheurs de la vertu, La Nouvelle Justine dans Euvres, t. II, Paris, Gallimard (Bibliothèque de la Pléiade) 1995, [M. Delon (éd.)]

SADE, Juliette dans Euvres, t. III, Paris, Gallimard (Bibliothèque de la Pléiade) 1998, [M. Delon (éd.)], p. 179-1262.

SÉVIGNÉ, Mme de, Correspondance, Paris, Gallimard (Bibliothèque de la Pléiade), 1972-1978, 3 vol. [R. Duchêne et J. Duchêne (éds.)].

VARENNE, Jacques de, Mémoires du chevalier de Ravanne, Aux dépends de la Compagnie, 1782, 4 vol.

VOLTAIRE, Candide, Paris, Librairie Générale Française (Le Livre de poche), 1995, [Sylviane Léoni (éd.)].

VOLTAIRE, La Princesse de Babylone dans Romans et Contes, Paris, Gallimard (Bibliothèque de la Pléiade), [1768] 1954 [R. Groos (éd.)], p. 366-432.

BERNARD, Michel, «Madame Bovary ou le danger des sucreries », Romantisme, n¹03, 1999, p. 41-51. 
BERNIER, Marc André, Libertinage et figures du savoir. Rhétorique et roman libertin dans la France des Lumières (1734-1751), Québec/Paris, Presses de l'Université Laval / L'Harmattan, (Les Collections de la République des Lettres), 2001.

BOKOBZA KAHAN, Michèle, Libertinage et Folie dans le roman du $18^{e}$ siècle, Louvain, Éditions Peeters (La République des Lettres), 2000.

BONNET, Jean-Claude, «Le réseau culinaire dans l'Encyclopédie», Annales. Économies, sociétés, civilisations, vol. 31, $\mathrm{n}^{\circ} 5,1976, \mathrm{p} .891-914$.

BRAUDEL, Fernand, Civilisation matérielle, économie et capitalisme, XVe-XVIIIe siècle, t. 1, Les structures du quotidien : le possible et l'impossible, Paris, Armand Colin, 1979.

COE, Sophie D. et COE, Michael D., Généalogie du chocolat, Paris, Éditions Abbeville, 1998. CORBIN, Alain, Le miasme et la jonquille, Paris, Champs Flammarion, [1982] 2016.

DELON, Michel, " Le détail et l'histoire », dans Claire JAQUIER, Florence LOTTERIE et Catriona SETH (éds.), Destins romanesques de l'émigration, Paris, Desjonquères, 2007, p. 158168.

DELON, Michel (éd.), Dictionnaire européen des Lumières, Paris, Presses Universitaires de France, 1997.

DIDIER, Béatrice, Sade : une écriture du désir, Paris, Denoël / Gonthier, 1976.

DUECK Evelyn et VUILLEMIN Nathalie (éds.), « Der Augen Blödigkeit », Sinnestäuschungen, Trugwahrnehmungen und visuelle Epistemologie im 18. Jahrhundert, Heidelberg, Winter, 2016.

DUECK Evelyn et VUILLEMIN Nathalie (éds.), Entre l'œil et le monde: dispositifs d'une nouvelle épistémologie visuelle dans les sciences de la nature (1740-1840), éd. Épistémocritique, 2017, en ligne. URL : https://epistemocritique.org/entre-loeil-monde-dispositifs-dune-nouvelleepistemologie-visuelle-sciences-de-nature-1740-1840/

EVANS, Michael R., Inventing Eleanor. The Medieval and Post-Medieval Image of Eleanor of Aquitaine, Londres / Oxford, Bloomsbury, 2014.

HERRERO Isabel et VAZQUEZ Lydia, « Types nationaux européens dans des œuvres de fiction françaises (1750-1789) », Dix-huitième Siècle, n²5, 1993, p. 115-127.

HOFFMANN, Kathryn A., « Palimpsests of Knowledge, Feast of Words : Antoine Furetière's Dictionnaire universel », Cahiers du dix-septième - An Interdisciplinary Journal, Vol. VII, n 1 , 1997, p. 47-59.

KOZUL, Mladen, Le Corps dans le monde. Récits et espaces sadiens, Louvain, Peeters, 2005.

LAFON, Henri, Les Décors et les choses dans le roman français du dix-huitième siècle de Prévost à Sade, Oxford, Voltaire Foundation, 1992.

LEVIN, D. M., Modernity and the Hegemony of Vision, Berkeley, University of California Press, 1993.

PATERNOTTE, Stéphanie et LABRUDE, Pierre, «Le chocolat dans quelques ouvrages français de pharmacie et de médecine des $\mathrm{XVII}^{\mathrm{e}}, \mathrm{XVIII}^{\mathrm{e}}$ et XIX ${ }^{\mathrm{e}}$ siècles. Ses effets fastes et néfastes avérés ou supposés », Revue d'histoire de la pharmacie, vol. 91, n³38, 2003, p. 197-210.

PEETERS, Alice, «Boire le chocolat», Terrain , $\mathrm{n}^{\circ} 13$, octobre 1989, URL: http://journals.openedition.org/terrain/2959.

PEETERS, Alice, «Controverses sur les vertus du cacao et les manières de préparer le chocolat (XVI $-\mathrm{XVIII}{ }^{\mathrm{e}}$ siècle) », Journal d'agriculture traditionnelle et de botanique appliquée, vol. 26, n³-4, Juillet-décembre 1979, p. 201-216.

PUCCINI-DELBEY, Géraldine, Le Débat des cinq sens de l'Antiquité à nos jours, Pessac, PUB, 2014.

Topiques, Études satoriennes, 5, 2020, Le Boire et le manger, https://journals.uvic.ca/index.php/sator/index 
RAMBOURG, Patrick, «De la consommation des boissons exotiques aux XVII et XVIII' siècles ", Thé, café ou chocolat? Les boissons exotiques à Paris au XVIII siècle, catalogue de l'exposition au Musée Cognacq-Jay, Paris musées, 2015, p. 47-61.

RIPOLL, Élodie, Penser la couleur en littérature. Explorations romanesques des Lumières au réalisme, Paris, Classiques Garnier (Confluences), 2018.

RUSTIN, Jacques, "Définition et explicitation du roman libertin des Lumières ", Travaux de linguistique et de littérature, XVI, 2, 1978, p. 26-34.

$\mathrm{SCHÖCH}$, Christof, La Description double dans le roman des Lumières, Paris, Classiques Garnier (L'Europe des Lumières), 2011.

SÉRET, Guillaume, «Des porcelaines pour sublimer le goût», Thé, café ou chocolat? Les boissons exotiques à Paris au XVIII siècle, catalogue de l'exposition au Musée Cognacq-Jay, Paris musées, 2015, p. 85-95.

SMITH, Mark M., Sensory History, Oxford \& New York, Berg, 2007.

STALNAKER, Joanna, The Unfinished Enlightenment. Description in the Age of the Encyclopedia, Ithaca \& Londres, Cornell UP, 2010.

CHARRIERE, Isabelle de, Euvres complètes d'Isabelle de Charrière / Belle de Zuylen, Amsterdam, van Oorschot, 1979-1984, 10 vols. (éds. Jean-Daniel CANDAUX, C.P. COURTNEY, Pierre H. DUBOIS, Simone DUBOIS-DE BRUYN, Pierre THOMPSON, Jeroom VERCRUYSSE ET Dennis M. WOOD).

Belle de Zuylen / Isabelle de Charrière. Éducation, création, réception, VAN DIJK, Suzan, COSSY, Valérie, MOSER-VERREY, Monique, VAN STRIEN-CHARDONNEAU, Madeleine (eds.), Amsterdam / New York, Rodopi (Coll. Faux-Titre, 276), 2006.

CITTON, Yves, « Les spectres de la multiplicité. La littérature du XVIII ${ }^{\mathrm{e}}$ siècle revisitée depuis ses dehors ", dans Christie McDONALD et Susan Rubin SULEIMAN (eds.), French Global. Une nouvelle perspective sur l'histoire littéraire, Paris, Classiques Garnier, 2014, p. 551-576, en ligne : https://hal.archives-ouvertes.fr/hal-01373195/document [cons. 2-3-2021]).

Écriture de la ruse, Elzbieta GRODEK, (ed.), Amsterdam / Atlanta, Rodopi (Coll. Faux-Titre, 190), 2000.

JONGBLOED, Hein H. et VAN DIJK, Suzan, « De vier “nieuwe” brieven van Belle van Zuylen », Tirade, 2012, no 56, p. 91-99.

KLOEK, Joost et MIJNHARDT, Wijnand, Dutch Culture in a European Perspective 2; 1800; Blueprints for a National Community, Londres, Palgrave Macmillan, 2004.

MOSER-VERREY, Monique, Isabelle de Charrière, salonnière virtuelle: Un itinéraire d'écriture au XVIII e siècle, Paris, Hermann, 2013.

NOORDERVLIET, Nelleke, Volg je eigen weg, postface à Belle VAN ZUYLEN, De Edelman (trad. du Noble par Rosalien VAN WITSEN), Amsterdam, Van Oorschot, 2013, p. 35-49.

PELCKMANS, Paul, «Adieu Poes que je chéris, quoiqu'il me mette de mauvaise humeur.... Isabelle de Charrière et Willem-René van Tuyll », dans VAN DIJK et al., Belle de Zuylen / Isabelle de Charrière. Éducation, création, réception. Amsterdam / New-York, Rodopi (Coll. Faux-Titre, 276), 2006, p. 69-84.

REID, Martine, « Dilemme, ou la condition des femmes », dans VAN DIJK et al., Belle de Zuylen / Isabelle de Charrière. Éducation, création, réception, Amsterdam / New York, Rodopi, (Coll. Faux-Titre, 276), 2006, p. 167-174.

VAN DEN BERGH, Greetje, Ik heb geen talent voor ondergeschiktheid. Belle van Zuylen in briefwisseling met Constant d'Hermenches, James Boswell en Werner C.W. van Pallandt. Amsterdam, Van Oorschot, 1987.

Topiques, Études satoriennes, 5, 2020, Le Boire et le manger, https://journals.uvic.ca/index.php/sator/index 
VAN DIJK, Suzan, «Topoi et généricité : inventaire et classement des topoi présentés », dans Suzan VAN DIJK et al., (éds.), Féminités et masculinités dans le texte narratif avant 1800. La question du 'gender', Louvain, Peeters (Coll. La République des Lettres, 6), 2002, p. 443-467.

VAN DIJK, Suzan, "Belle de Zuylen et les 'talents' des Hollandaises », in Cahiers Isabelle de Charrière / Belle de Zuylen Papers, 2010, $\mathrm{n}^{\circ}$ 5, p. 64-74.

VAN DIJK, Suzan et SCHOUTEN, Maria, « Numériser les lettres de Belle de Zuylen: Un regard plus précis sur les rapports familiaux », in Cahiers Isabelle de Charrière/Belle de Zuylen Papers, 2013, no 8, p. 24-44.

VAN DIJK, Suzan, « Amitié, solidarité et entraide féminines : Spécificités d'auteurs femmes ? », Topiques. Études Satoriennes, éd. Hélène Cazes, 2015/1, http://journals.uvic.ca/index.php/sator/article/view/12633.

VAN DIJK, Suzan, en collaboration avec SCHOUTEN, Maria, « La correspondance d'Isabelle de Charrière (1740-1805). Survie et destruction des lettres comme thématiques de l'épistolière », dans Matthieu MAGNE (éd.), Prendre la plume des Lumières au Romantisme, Clermont-Ferrand, Presses Universitaires Blaise Pascal, 2019, p. 149-164.

VAN DIJK, Suzan, « Belle van Zuylen: schrijfster van adel, over de adel. Haar correspondentie digitaal beschikbaar », à paraître dans Virtus, Jaarboek voor Adelsgeschiedenis. 2021.

VAN STRIEN-CHARDONNEAU, Madeleine, "Isabelle de Charrière, pédagogue », dans VAN DIJK et al., 2006, Suzan VAN DIJK et al., Belle de Zuylen / Isabelle de Charrière. Education, création, réception, Amsterdam / New York, Rodopi (Coll. Faux-Titre, 276), 2006, p. 49-68.

VISSIERE, Isabelle et VISSIERE, Jean-Louis (éds.), Isabelle de Charrière. Une liaison dangereuse : Correspondance d'Isabelle de Charrière avec Constant d'Hermenches, 1760-1776, Paris, La Différence, 1991.

WENT-DAOUST, Yvette, «La place des Lettres neuchâteloises dans le roman épistolaire du XVIIIe siècle », in Doris JAKUBEC et Jean-Daniel Candaux (éds)., Une Européenne : Isabelle de Charrière en son siècle, Neuchâtel, Eds. Gilles Attinger, 1994, p. 187-196.

Topiques, Études satoriennes, 5, 2020, Le Boire et le manger, https://journals.uvic.ca/index.php/sator/index 